\title{
Pedicle-Lengthening Osteotomy for the Treatment of Lumbar Spinal Stenosis: Pre-Clinical Study of Novel Orthopedic Devices
}

\author{
DOI: $10.17691 / \mathrm{stm} 2018.10 .2 .04$
}

Received January 18, 2018

S.G. Mlyavykh, MD, PhD, Director of the Institute of Traumatology and Orthopedics';

A.E. Bokov, MD, PhD, Head of the Neurosurgery Department, Institute of Traumatology and Orthopedics";

K.S. Yashin, MD, Junior Researcher, Laboratory of Super-Resolution Microscopy and Gene Technologies, Institute of Biomedical Technologies ${ }^{1 ;}$; Neurosurgeon, Neurosurgery Department, Institute of Traumatology and Orthopedics ${ }^{1}$;

N.N. Karyakin, MD, DSc, Rector';

D.G. Anderson, MD, Professor, Departments of Orthopaedic and Neurological Surgery²; Clinical Director of the Spine Section, Orthopaedic Research Laboratory²

1Privolzhsky Research Medical University, 10/1 Minin and Pozharsky Square, Nizhny Novgorod, 603005, Russia; ${ }^{2}$ Thomas Jefferson University, 130 S., 9th Street, Philadelphia, PA, 19107, USA

The growing number of surgical interventions in patients with spinal degenerative diseases and spinal cord injuries necessitate the development and implementation of innovative technologies for spinal surgery. Successful pre-clinical tests assessing the safety and reliability of the proposed devices are a major step towards further routine use of the novel technologies.

In this report, we describe preclinical studies on screw implants designed to length vertebral pedicles in the lumbar spine and provide indirect decompression of the nerve structures by increasing the transverse size of the spinal canal and intervertebral foramen. The results demonstrated high reliability of the developed device, its potential effectiveness in eliminating lumbar spinal stenosis, and only minor negative effects on the lumbar spine function. Altogether, our study made it possible to start a pilot clinical project on using this technology in patients with symptomatic lumbar spinal stenosis.

Key words: lumbar stenosis; vertebral pedicle-lengthening; pedicle screw implants; vertebral surgery.

\section{Introduction}

Degenerative disorders of the spine are common causes of disability among the working-age population [1]. Surgical treatment has been increasingly used in patients of this category [2,3], which is associated, among other factors, with the active implementation into clinical practice of minimally invasive technologies $[4,5]$ capable of safe the normal anatomy of the adjacent soft tissues. Since recently, the indications for surgical treatment of various spine disorders have been expanded due to the reduced risk of surgical complications, blood loss, infections, and "physiological stress".

In turn, the increase in the number of surgical interventions and the continuing dissatisfaction with their results create a strong motivation for the development and implementation of innovative technologies and methods of surgical treatment. Thus, since recently there has been a notable growth of the market of implants and surgical instruments for spine surgery [5].
Orthopedic surgeons and neurosurgeons are taking an ever more active part in their development. The daily contact with patients allows them to propose improvements and new methods of surgical interventions $[6,7]$. Moreover, they can and do contribute to success of preclinical studies designed to test the safety and reliability of novel methods and devices.

In the development of spinal implants, a static and dynamic testing is used to assess their stability and biomechanical properties and to predict their functioning after the implantation. Preservation of normal anatomy and physiology of the spine is a key factor that determines the success and clinical significance of the treatment.

In this article, the results of preclinical trials of the newly developed spinal implants [8, 9] for minimally invasive correction of lumbar spine stenosis are presented. This technology is based on bilateral osteotomy of the pedicles of the vertebrae followed by their subsequent lengthening using special screw implants (Figure 1). This manipulation results in an

Corresponding author: Sergey G. Mlyavykh, e-mail: serg.mlyavykh@gmail.com 


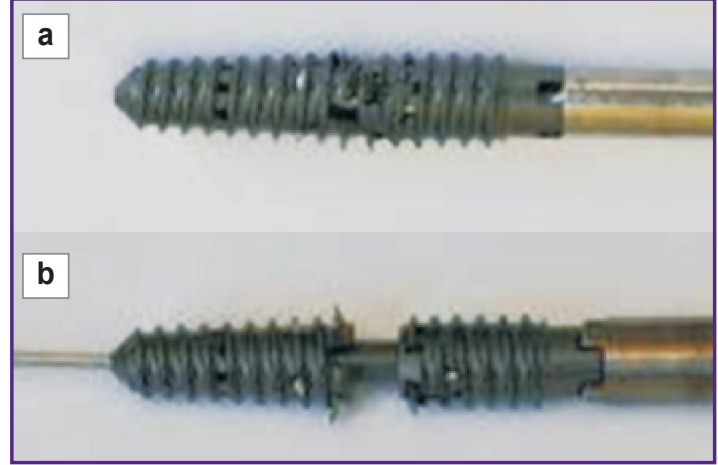

Figure 1. The appearance of the pedicle screw implant before (a) and after (b) its lengthening

indirect decompression of the neural structures due to a widening of the vertebral canal and intervertebral foramina.

The implant design used in this technology relates to Class 2 medical products per classification of the FDA [10] and to Class $2 b$ as defined by the Ministry of Healthcare of the Russian Federation of June 6 , 2012 "On approval of the nomenclature classification of medical devices". In the same group, there are most orthopedic implants, for example, screws for transpedicular fixation.

Preclinical trials of these devices are required to include: 1) identification of possible complications resulting from the interaction of the implant with the surrounding tissues (animal testing); 2) testing the mechanical strength of the structure (bench tests); and 3) in vitro assessment of biomechanical properties and stability of the spine after the implantation (performance tests).

\section{Animal testing}

Animal studies are conducted to identify possible complications caused by the interaction between the implant and the surrounding tissues, to assess the process of tissue healing and implant wear, and also to look into possible changes in the neural structures. These studies are expected to provide the results essential for the further clinical use of the implant. For example, in the intervertebral disc arthroplasty it advisable to evaluate the spine function, this, however, may be difficult after extensive surgical interventions and subsequent animal restraint.

The choice of the animal model depends, to some extent, on the implant design, but in most cases (for arthroplasty of the intervertebral disc, posterior transpedicular dynamic stabilization, interstitial spacers and facet joints), animals such as sheep, goats, large dogs are preferable [11].

As an animal model in this preclinical study on the osteotomy technology and vertebral pedicle-lengthening,
Yorkshire cross pigs were selected. The choice was prompted by the specific anatomical structure of their vertebrae, which allowed us to perform osteotomy using the standard surgical instruments without the need to adjust the size and shape of clinically used surgical tools to the purpose of preclinical testing.

Three animals were included in the study; they underwent bilateral osteotomy of the $L_{4}$-vertebrae pedicles with their subsequent lengthening by $3 \mathrm{~mm}$ under general anesthesia and X-ray guidance. In the postoperative period, the animals were evaluated for their behavior and functional status. In the early postoperative period and throughout the later observation, no signs of neurologic deficit were noted. According to the approved protocol, the animals were withdrawn from the experiment 3 months later. The subsequent morphological analysis of the osteotomy zone in all three animals showed: a) the formation of bone tissue (fusion) in the osteotomy zone; and b) no signs of iatrogenic heterotopic ossification.

This animal study allowed us to conclude on good biocompatibility of the implant, preservation of the spinal functions after its implantation and fast formation of bone tissue in the operated area.

\section{Bench testing}

Bench tests are conducted to quantify the static and dynamic characteristics of various spinal implants and their compliance with the standards of the American Society for Testing and Materials (ASTM) and the International Organization for Standardization (ISO). The in vitro studies using simplified loading protocols are not intended to simulate a multi-component load on the spine (fatigue load method). Specific requirements for mechanical testing depend on the design, the implant material, the way of attachment to the spine, and the type of clinical use [12]. In accordance with these requirements, the relevant study design has been selected.

For spine fixation, structures consisting of several elements are most often used. The main purpose of this design is to ensure steadiness of the fixed vertebral motion segments (VMS) under physiological loads for the entire time needed for spondylodesis formation. At present, the ASTM F1717 and ISO 12189 standards [13-17] are used to assess the mechanical strength of prefabricated structures for posterior spine stabilization. These standards differ from each other: in the first case, the vertebrectomy model is used, and in the second case, it is the physiological model of the spine with instrumentation (Figure 2).

After the implant is placed onto the spine model, the fatigue tests can be conducted. In these, the implant is evaluated at the starting loads where the maximum fatigue load is $2000 \mathrm{~N}$, which is close to the physiological compression load on the lumbar discs [18]. As the test continues, the loads gradually increase until the implant structure is destroyed. 


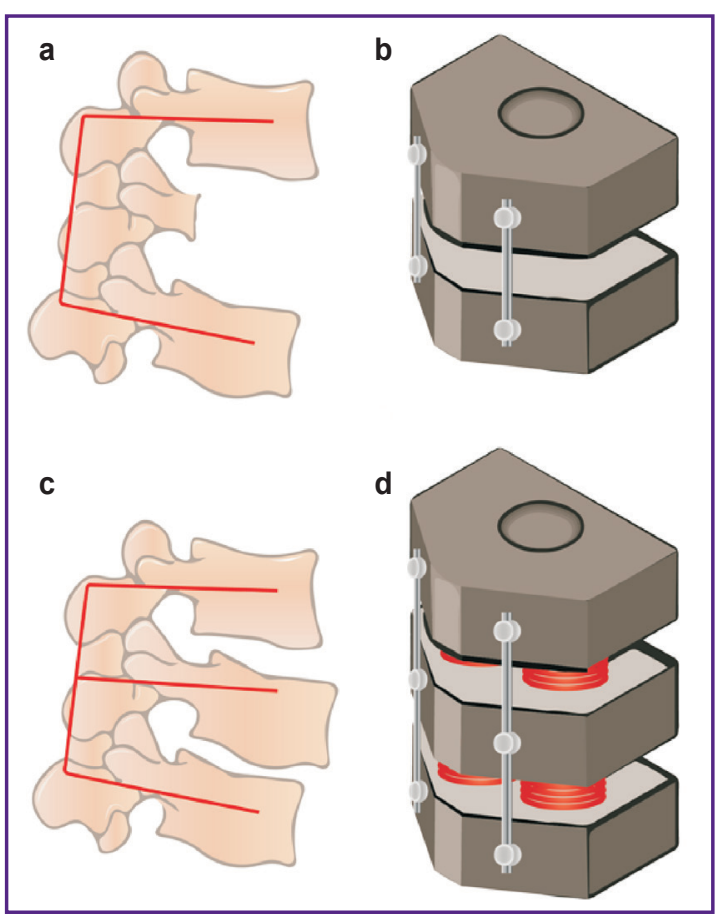

Figure 2. Currently available experimental models for testing the mechanical characteristics of the posterior spine stabilization devices:

(a), (b) vertebrectomy model; (c), (d) physiological models of the spine with instrumentation according to ASTM F1717 and ISO 12189 , respectively

The ASTM F1717 standard is most suitable for rigid structures that are designed to provide a rigid fixation and the subsequent formation of the bone block, while ISO 12189 relates largely to the implants intended to facilitate the process of spondylodesis and preserve the limited mobility in spinal segment [15].

In this study, the purpose of the implant placing was to fix two bone components (vertebral body and vertebral arch) within the same bone during the healing process. Accordingly, we chose the commonly accepted standards for testing of screws for transpedicular fixation, namely, ASTM F2193 (static cantilever bending), ASTM F543-07 (screw insertion push-out), and ASTM F54307 (dynamic bending, $3 \mathrm{~mm}$ expansion). The purpose of using the above standards was to evaluate the various strength characteristics of the proposed implant (screw).

\section{Performance testing}

Biomechanical study. Research into biomechanics of the spine under in vitro conditions using various anatomical models is now common [19]. In most cases, such a study aims at analyzing the mobility of one or several VMS under simulated loads; in these experiments, either a single segment or multisegment models of the spine are employed. There are a large number of functional testing options with various force directions and magnitudes. In vitro tests and assessments of the spine biomechanical characteristics, following spine instrumentation, have a number of technical limitations like the changing mechanical properties of the anatomical samples with time, a great degree of variability between the samples, and the impossibility of testing several implants types in this fashion.

There are three major protocols for biomechanical testing of a spinal segment: for flexibility, for stiffness, and a hybrid test. The most common is the flexibility protocol [20], in which a known force is applied to the vertebral segment subjected to surgical intervention. The surgery outcome is evaluated by determining the degree of displacement before and after the surgery.

The rigidity protocol employs the opposite approach [21], in which the degree of segment displacement is tightly controlled, and the applied load is measured and then compared with the preoperative value. The physiological rationale behind this protocol is the requirement to preserve the preoperative range of motion necessary for restoring the daily patient's activity.

The hybrid protocol is recommended for studies on a specific section of the spine as a whole (for example, $\mathrm{Th}_{12}$ - sacrum). It includes three main stages [22]: 1) application unconstrained pure loads to intact segments of the spine (control); 2) application unconstrained pure loads to the treated segments of the spine (after implant placement) until it reaches the range of motions identical to the control values; and 3) statistical comparison of biomechanical indices between steps 1 and 2 . The analysis provides an assessment of the surgery-induced spine instability and the possible development of adjacent level syndrome. This protocol is based on the assumption that the spine is able to get adapted after the surgical instrumentation, restoring its natural range of motions.

The main goal of the proposed procedure for elongating the vertebral pedicles is to eliminate the compression of nerve roots while preserving the physiological range of motions in all lumbar segments. With that in mind, we selected a hybrid test protocol with instrumentation of 8 lumbosacral segments $L_{1}-S_{1}$ taken from donors (mean age $70 \pm 4$ years) [23, 24]. Just prior to the segment extraction, an X-ray examination was conducted to rule out possible anatomical defects. After thawing the samples and bringing them to room temperature, a dissection was performed while preserving the spine ligaments (anterior longitudinal, posterior longitudinal, interspinal and supraspinal ligaments, and ligamentum flavum) and, most importantly, the facet joints and their capsules.

Each sample was placed on the kinematic profiler device, where the upper $\left(L_{1}\right)$ and lower $\left(S_{1}\right)$ vertebrae were rigidly fixed using a rectangular frame using polyester-styrene resin (Bondo; 3M, USA), and embedded transfixing pins (Figure 3). The positioning 

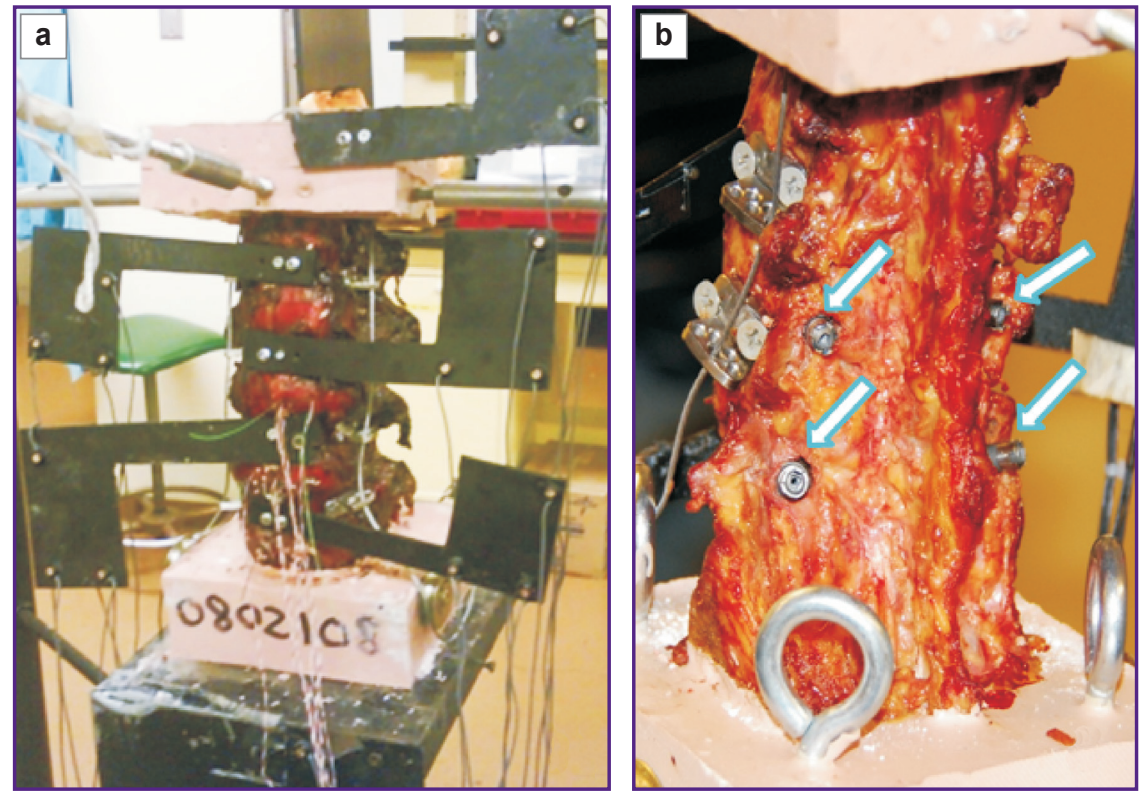

Figure 3. The experimental set for the study on spine biomechanics using an anatomical sample of the spine:

(a) installation equipped with Optotrak camera system; (b) the anatomical sample with the $L_{1}-S_{1}$ spine segments is installed in a kinematic profiler, which enables extending of vertebral pedicles at $\mathrm{L}_{4}$ and $\mathrm{L}_{5}$; arrows denote the implants placed after bilateral osteotomy of $\mathrm{L}_{4}$ and $\mathrm{L}_{5}$ pedicles

of the spinal segment was made in such a way that the axial plane of the $L_{3}-L_{4}$ disc was in the horizontal (most physiological) position. Each vertebral body was equipped with special plates that contained infrared LEDs (IRED) and allowed to monitor changes in the position of each vertebra using the Optotrak camera system (Model 3020; Northern Digital, Canada). The

Table 1

Range of motion (degrees) in the $L_{1}-S_{1}$ segment before and after the pedicle-lengthening (Mean $\pm 95 \% \mathrm{Cl}$ )

\begin{tabular}{|c|c|c|c|c|}
\hline $\begin{array}{c}\text { State } \\
\text { of the segment }\end{array}$ & Level & $\begin{array}{l}\text { Flexion/ } \\
\text { extension }\end{array}$ & $\begin{array}{l}\text { Bending } \\
\text { right/left }\end{array}$ & $\begin{array}{l}\text { Axial rotation } \\
\text { right/left }\end{array}$ \\
\hline Intact & \multirow{4}{*}{$\mathrm{L}_{3}-\mathrm{L}_{4}$} & $10.3 \pm 2.7$ & $11.6 \pm 2.9$ & $7.6 \pm 2.9$ \\
\hline $\mathrm{L}_{4}$ lengthening & & $9.9 \pm 4.3$ & $13.2 \pm 3.6$ & $7.8 \pm 4.4$ \\
\hline $\mathrm{L}_{5}$ lengthening & & $10.4 \pm 2.9$ & $12.2 \pm 3.9$ & $7.5 \pm 4.0$ \\
\hline $\mathrm{L}_{4}-\mathrm{L}_{5}$ lengthening & & $11.8 \pm 4.3$ & $12.9 \pm 2.6$ & $7.7 \pm 2.4$ \\
\hline Intact & \multirow{4}{*}{$\mathrm{L}_{4}-\mathrm{L}_{5}$} & $11.4 \pm 1.8$ & $12.0 \pm 2.5$ & $7.5 \pm 2.2$ \\
\hline $\mathrm{L}_{4}$ lengthening & & $13.2 \pm 3.2$ & $13.4 \pm 2.6$ & $8.9 \pm 3.3$ \\
\hline $\mathrm{L}_{5}$ lengthening & & $10.2 \pm 2.5$ & $11.0 \pm 3.5$ & $6.3 \pm 2.0$ \\
\hline$L_{4}-L_{5}$ lengthening & & $11.6 \pm 2.7$ & $12.2 \pm 3.1$ & $8.0 \pm 1.8$ \\
\hline Intact & \multirow{4}{*}{$\mathrm{L}_{5}-\mathrm{S}_{1}$} & $14.0 \pm 2.7$ & $10.0 \pm 1.8$ & $8.4 \pm 1.8$ \\
\hline $\mathrm{L}_{4}$ lengthening & & $15.1 \pm 2.9$ & $11.2 \pm 1.6$ & $9.1 \pm 2.1$ \\
\hline $\mathrm{L}_{5}$ lengthening & & $13.2 \pm 5.4$ & $9.8 \pm 4.7$ & $7.0 \pm 4.1$ \\
\hline$L_{4}-L_{5}$ lengthening & & $13.4 \pm 2.7$ & $12.1 \pm 2.6$ & $10.0 \pm 2.5$ \\
\hline Intact & \multirow{4}{*}{$\mathrm{L}_{3}-\mathrm{S}_{1}$} & $35.6 \pm 6.0$ & $33.6 \pm 6.2$ & $23.6 \pm 5.6$ \\
\hline $\mathrm{L}_{4}$ lengthening & & $39.3 \pm 5.1$ & $36.3 \pm 7.0$ & $26.3 \pm 2.7$ \\
\hline$L_{5}$ lengthening & & $38.2 \pm 4.7$ & $37.7 \pm 6.1$ & $25.7 \pm 4.2$ \\
\hline $\mathrm{L}_{4}-\mathrm{L}_{5}$ lengthening & & $33.7 \pm 7.6$ & $33.2 \pm 7.0$ & $21.5 \pm 5.1$ \\
\hline
\end{tabular}

testing protocol included an application of paired forces to segments $L_{1}-S_{1}$ in three directions: flexion/extension, left/right lateral bending, and left/right axial rotation. The pure moment force application was performed stepwise with a gradual increase (each step $0.25 \mathrm{~N} \cdot \mathrm{m}$ ) to the cephalad end of the spinal segment while the caudal end of the spinal segment was fixed in position until a moment of $10 \mathrm{~N} \cdot \mathrm{m}$ was achieved. During the test, the position of each vertebra in the dorsal segment was monitored individually.

At the first stage, all 8 segment samples were tested. Then 4 samples were tested after carrying out a bilateral pedicle-lengthening of the $L_{4}$ pedicles by $4.5 \mathrm{~mm}$, while the other 4 samples were tested after lengthening of the $L_{5}$ pedicles by $4.5 \mathrm{~mm}$. And then, all 8 samples were tested again after bilateral pedicle-lengthening on both $L_{4}$ and $L_{5}$. In all cases, lengthening was performed using the methodology developed for clinical applications.

In the functional in vitro testing, the main parameter of interest is the range of motions as a measure of VMS instability caused by the implant placement; in addition, such a measurement shows whether motions of the adjacent segments are restricted. The range of motions for the intact state and following the three pedicle-lengthening scenarios $\left(L_{4}, L_{5}\right.$, and $\left.L_{4}-L_{5}\right)$ for the three planes of motion at the $L_{3}-L_{4}, L_{4}-L_{5}, L_{5}-S_{1}$ levels and the overall $L_{3}-S_{1}$ segment are shown in Table 1 . The average range of motions for each type of pediclelengthening lies within a confidence interval of $95 \%$, indicating that there is no significant difference from the intact sample.

To assess the stability of the lumbar spine sample, we used the so-called load-displacement test. In this, the typical curves of the $L_{4}-L_{5}$ displacement under the moment of forces (in the flexion/extension mode) with a compression load of $400 \mathrm{~N}$ were similar between the 
intact pedicles and those after lengthening at the $L_{4}, L_{5}$, and $L_{4}-L_{5}$ levels (Figure 4).

Thus, the above experimentation allowed us to confirm that osteotomy of the vertebral pedicles and their subsequent lengthening with implants at one or more levels would not lead to the VMS instability or to the development of adjacent level syndrome.

Computer modeling. Currently, methods of computer modeling are increasingly used to study biomechanical factors affecting the spine function under normal and abnormal conditions including fixation devices and implants [25]. One of the most common is the finite element model analysis, which has been used by a number of authors $[26,27]$. In this method, a model of a vertebral segment with an intervertebral disk is created. The basic substance of the fibrous ring is simulated by the hyperelastic Mooney-Rivlin material, the nucleus pulposus - by an incompressible liquid, and the bone tissue of the vertebra - by the implant (isotropic) material [28] (Figure 5 (a)). In most studies, the model considers the rigid fixation of the lower vertebra $\left(L_{5}\right.$ or $\left.S_{1}\right)$, to which various loads are applied (either three-directional bending moments or combined loads) with simultaneous monitoring of changes in the range of motions and internal disk pressure in each VMS.

In the present study, the finite element method was used to:

evaluate changes in the cross-sectional area of the vertebral canal and the intervertebral foramina after the pedicle-lengthening;

quantify the effect of this procedure on the biomechanical characteristics of the lumbar segment.

This $3 \mathrm{D}$ model of the $\mathrm{L}_{3}-\mathrm{S}_{1}$ segment, containing

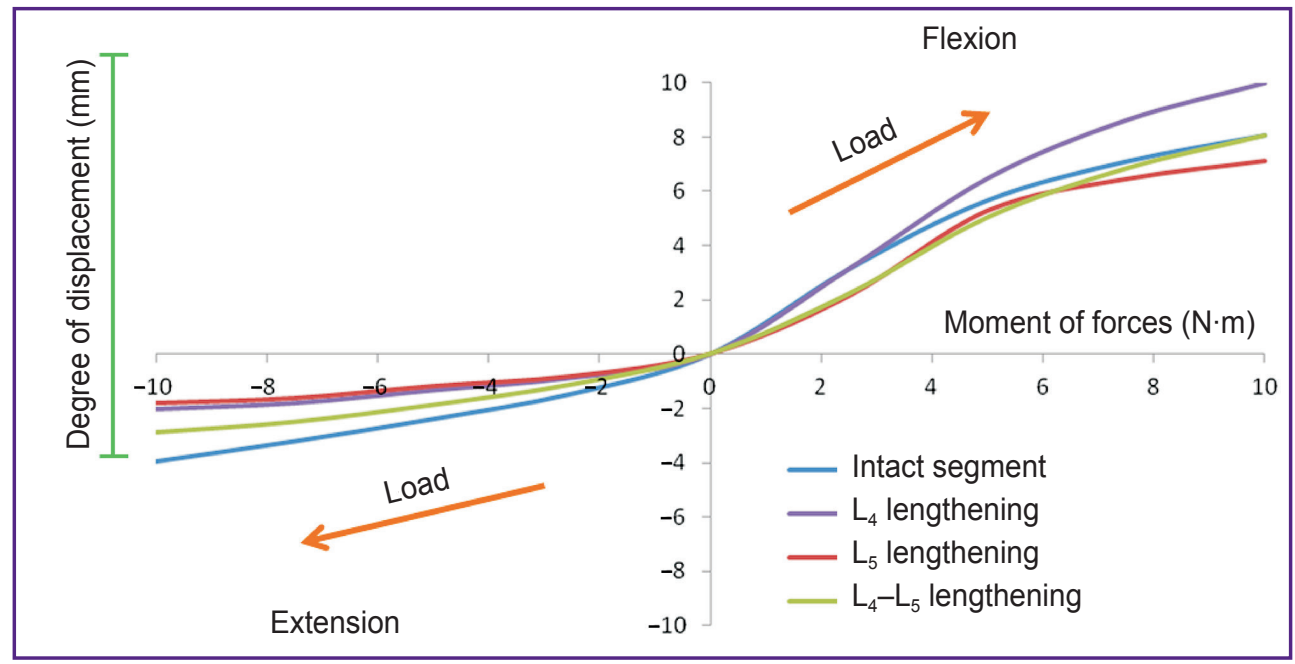

Figure 4. The load-displacement curves obtained with the $L_{4}-L_{5}$ implant model for flexing/ extending at compression load of $400 \mathrm{~N}$

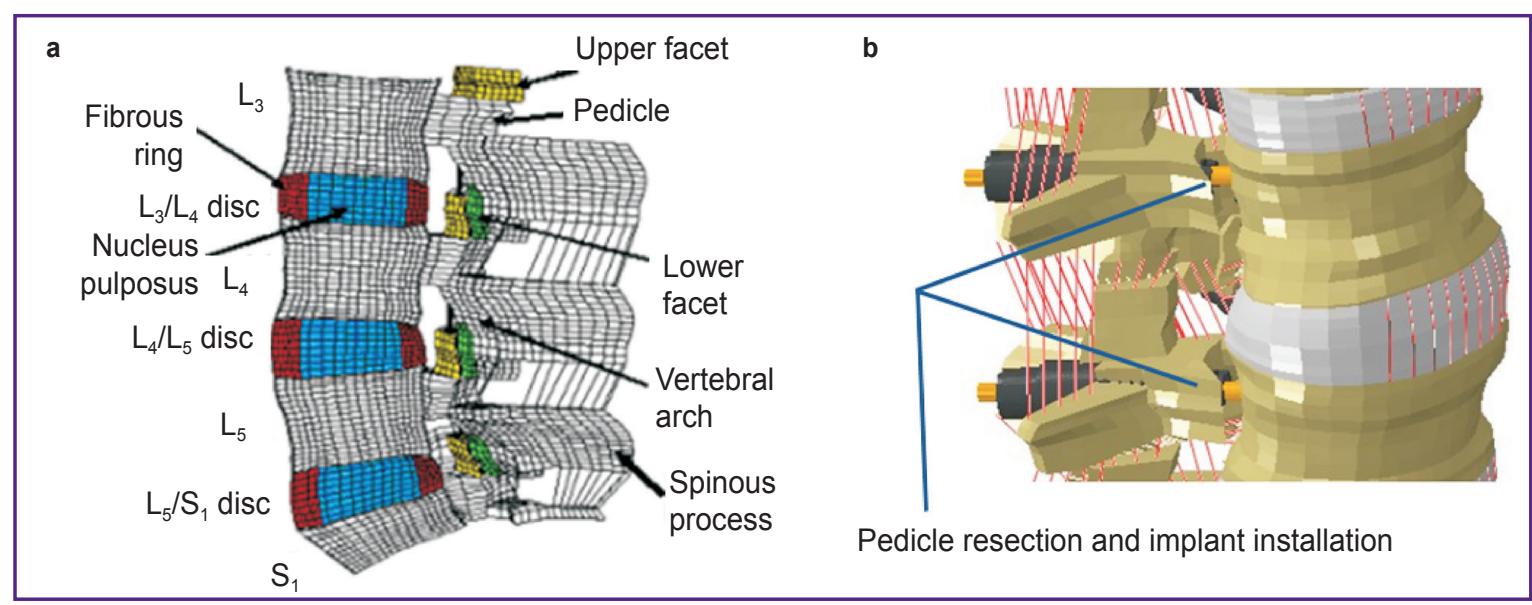

Figure 5. Finite element computer simulation for evaluating of new spinal implants:

(a) computer model of $\mathrm{L}_{3}-\mathrm{S}_{1}$ segment; (b) simulated pedicle resection adjacent to the vertebral bodies and the following installation of an extending implant 
Table 2

Increased cross-sectional areas (\%) of the spinal canal and the intervertebral foramen at levels $L_{3}-L_{4}, L_{4}-L_{5}$, and $L_{5}-S_{1}$ upon simulated pedicle-lengthening (by 2,3 , or $4.5 \mathrm{~mm}$ ) using the finite element analysis

\begin{tabular}{|c|c|c|c|c|c|c|c|}
\hline \multirow{2}{*}{$\begin{array}{c}\text { State } \\
\text { of the segment }\end{array}$} & \multirow{2}{*}{ Level } & \multicolumn{3}{|c|}{ Spinal canal $\left(\mathrm{mm}^{2}\right)$} & \multicolumn{3}{|c|}{ Intervertebral foramen $\left(\mathrm{mm}^{2}\right)$} \\
\hline & & 2 & 3 & 4.5 & 2 & 3 & 4.5 \\
\hline \multirow{3}{*}{$\mathrm{L}_{4}$ lengthening } & $\mathrm{L}_{3}-\mathrm{L}_{4}$ & 3.7 & 5.7 & 8.9 & 12.6 & 19.2 & 28.3 \\
\hline & $\mathrm{L}_{4}-\mathrm{L}_{5}$ & 10.8 & 15.3 & 22.2 & 7.4 & 11.6 & 18.0 \\
\hline & $\mathrm{L}_{5}-\mathrm{S}_{1}$ & 0.0 & 0.1 & 0.2 & 0.0 & 0.0 & 0.0 \\
\hline \multirow{3}{*}{$\mathrm{L}_{5}$ lengthening } & $\mathrm{L}_{3}-\mathrm{L}_{4}$ & 0.1 & 0.1 & 0.2 & -0.1 & 0.0 & 0.0 \\
\hline & $\mathrm{L}_{4}-\mathrm{L}_{5}$ & 4.4 & 6.8 & 10.8 & 13.0 & 19.6 & 28.8 \\
\hline & $\mathrm{L}_{5}-\mathrm{S}_{1}$ & 10.7 & 15.3 & 22.3 & 6.5 & 10.9 & 18.8 \\
\hline \multirow{3}{*}{$\mathrm{L}_{4}-\mathrm{L}_{5}$ lengthening } & $\mathrm{L}_{3}-\mathrm{L}_{4}$ & 3.6 & 5.6 & 8.8 & 12.4 & 18.7 & 27.9 \\
\hline & $\mathrm{L}_{4}-\mathrm{L}_{5}$ & 12.9 & 19.5 & 29.6 & 17.3 & 26.4 & 40.4 \\
\hline & $\mathrm{L}_{5}-\mathrm{S}_{1}$ & 10.5 & 15.0 & 21.6 & 6.4 & 10.7 & 18.4 \\
\hline
\end{tabular}

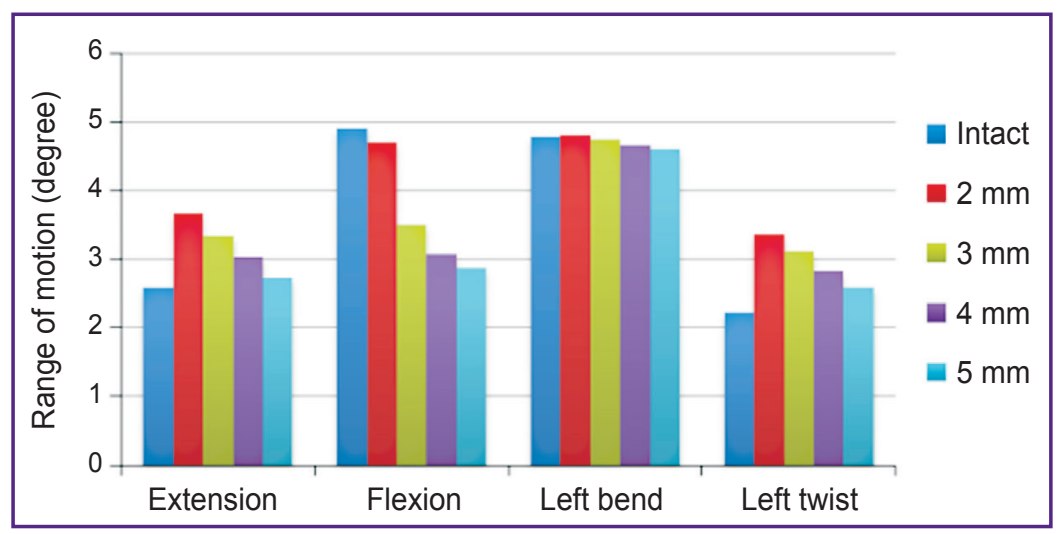

Figure 6. Changes in the range of motions in the $L_{4}-L_{5}$ segment upon pedicle-lengthening (by $2,3,4$, or $5 \mathrm{~mm}$ ) as simulated by the finite element method

27,540 elements and 32,946 nodes, was described earlier in a number of reports [29-32]. Using the prototypes, a 3D computer model of the implant was created. Further, the software of the existing lumbar spine model was modified so to imitate a one-level pedicle-lengthening at the $L_{4}$ or $L_{5}$ levels and a two-level lengthening at the $L_{4}-L_{5}$ segment (Figure $5(b)$ ). Then, the area of the vertebral canal cross-section and the intervertebral foramen was measured before and after the bilateral pedicle-lengthening by 2,3 , and $4.5 \mathrm{~mm}$.

Using the integrated software, we were able to measure the areas of the vertebral canal and intervertebral foramen at the $L_{3}-L_{4}, L_{4}-L_{5}$, and $L_{5}-S_{1}$ levels before and after bilateral pedicle-lengthening by 2,3 , and $4.5 \mathrm{~mm}$ (Table 2). It can be seen that the increase in the cross-sectional area of both the spinal canal and the intervertebral foramen depends on the size of the pedicle-lengthening. At the level of the $L_{4}-L_{5}$ disc, when the $L_{4}$ pedicles are extended by 2,3 , or $4.5 \mathrm{~mm}$, the cross-sectional area of the spinal canal increases by

\section{Conclusion} elements.
10,15 , or $22 \%$, respectively, and the both pedicle-lengthening in $L_{4}-L_{5}$ resulted in the widening of the spinal canal by 13,20 , or $30 \%$, respectively. When both $L_{5}$ pedicles are extended by 2,3 , or $4.5 \mathrm{~mm}$, the cross-sectional area of the intervertebral foramen is increased by 13,20 , or $29 \%$, respectively, and with simultaneous lengthening of both pedicles $L_{4}$ and $L_{5}$ by 17,26 , or $40 \%$, respectively.

Also, the finite element analysis was used to calculate the range of motions for different types of vertebral pediclelengthening at the $\mathrm{L}_{3}-\mathrm{L}_{4}, \mathrm{~L}_{4}-\mathrm{L}_{5}, \mathrm{~L}_{5}-\mathrm{S}_{1}$, and $L_{3}-S_{1}$ levels. The obtained data were then compared with the observed range of motions in experiments on anatomical samples. In all cases, the range of motions predicted by the model was within or close to $95 \%$ confidence intervals of the mean values obtained by direct measurements. This result further validated the computer model used in this study.

When assessing the range of motions, we found that upon increasing the pedicle lengths there was no restriction of motions in the cases of lateral flexion or rotation; the minimal restriction was observed in the case of extension, and a marked restriction occurred in the case of flexion (Figure 6).

Thus, the methods of computer modeling allowed us to estimate the increase in the spinal canal diameter and confirm the earlier findings of biomechanical study that the implants in question would not cause adjacent level disease.

In this report, the results of preclinical testing of the innovative use of implants for vertebrate pediclelengthening in degenerative lumbar stenosis are presented. The study demonstrates the advantages of the proposed technology that is based on pedicle screw implants, the minimally invasive osteotomy, and subsequent lumbar vertebrae lengthening. The developed system is reliable and potentially effective in eliminating the lumbar spine stenosis; it is free of any significant negative effect on the vertebral anatomical

Acknowledgments. The authors are grateful to A. Kiapo, D.B. Spencineru, L.A. Ferrara, V.K. Goel for their assistance in carrying out the experiments presented in this article.

Research funding and conflict of interest. The study was not funded by any source, and there is no conflict of interest related to this study. 


\section{References}

1. Global Burden of Disease Study 2013 Collaborators; Vos T., Barber R.M., Bell B., Bertozzi-Villa A., Biryukov S., Bolliger I., Charlson F., Davis A., Degenhardt L., Dicker D., Duan L., Erskine H., Feigin V.L., Ferrari A.J., Fitzmaurice C., Fleming T., Graetz N., Guinovart C., Haagsma J., Hansen G.M., Hanson S.W., Heuton K.R., Higashi H., Kassebaum N., Kyu H., Laurie E., Liang X., Lofgren K., Lozano R., Maclntyre M.F., Moradi-Lakeh M., Naghavi M., Nguyen G., Odell S., Ortblad K., Roberts D.A., Roth G.A., Sandar L., Serina P.T., Stanaway J.D., Steiner C., Thomas B., Vollset S.E., Whiteford H., Wolock T.M., Ye P., Zhou M., Ãvila M.A., Aasvang G.M., Abbafati C., Abbasoglu Ozgoren A., AbdAllah F., Abdel Aziz M.I., Abera S.F., Aboyans V., Abraham J.P., Abraham B., Abubakar I., Abu-Raddad L.J., Abu-Rmeileh N.M., Aburto T.C., Achoki T., Ackerman I.N., Adelekan A., Ademi Z., Adou A.K., Adsuar J.C., Arnlov J., Agardh E.E., Al Khabouri M.J., Alam S.S., Alasfoor D., Albittar M.I., Alegretti M.A., Aleman A.V., Alemu Z.A., Alfonso-Cristancho R., Alhabib S., Ali R., Alla F., Allebeck P., Allen P.J., AlMazroa M.A., Alsharif U., Alvarez E., Alvis-Guzman N., Ameli O., Amini H., Ammar W., Anderson B.O., Anderson H.R., Antonio C.A., Anwari P., Apfel H., Arsenijevic V.S., Artaman A., Asghar R.J., Assadi R., Atkins L.S., Atkinson C., Badawi A., Bahit M.C., Bakfalouni T., Balakrishnan K., Balalla S., Banerjee A., BarkerCollo S.L., Barquera S., Barregard L., Barrero L.H., Basu S., Basu A., Baxter A., Beardsley J., Bedi N., Beghi E., Bekele T., Bell M.L., Benjet C., Bennett D.A., et al. Global, regional, and national incidence, prevalence, and years lived with disability for 301 acute and chronic diseases and injuries in 188 countries, 1990-2013: a systematic analysis for the Global Burden of Disease Study 2013. Lancet 2015; 386(9995): 743800, https://doi.org/10.1016/s0140-6736(15)60692-4.

2. Deyo R.A., Mirza S.K. Trends and variations in the use of spine surgery. Clin Orthop Relat Res 2006; 443: 139-146.

3. Rajaee S.S., Bae H.W., Kanim L.E., Delamarter R.B. Spinal fusion in the United States: analysis of trends from 1998 to 2008. Spine 2012; 37(1): 67-76, https://doi.org/10.1097/ brs.0b013e31820cccfb.

4. Smith Z.A., Fessler R.G. Paradigm changes in spine surgery - evolution of minimally invasive techniques. Nat Rev Neurol 2012; 8(8): 443-450, https://doi.org/10.1038/ nrneurol.2012.110.

5. Spetzger U., Schilling A.V., Winkler G., Wahrburg J., König A. The past, present and future of minimally invasive spine surgery: a review and speculative outlook. Minim Invasive Ther Allied Technol 2013; 22(4): 227-241, https://doi. org/10.3109/13645706.2013.821414.

6. Babu M.A., Heary R.F., Nahed B.V. Device innovation in neurosurgery. Neurosurgery 2012; 70(4):789-795, https://doi. org/10.1227/neu.0b013e318237a68b.

7. Amstutz H.C. Innovations in design and technology. The story of hip arthroplasty. Clin Orthop Relat Res 2000; 378: 23-30.

8. Mlyavykh S., Ludwig S.C., Mobasser J.-P., Kepler C.K., Anderson D.G. Twelve-month results of a clinical pilot study utilizing pedicle-lengthening osteotomy for the treatment of lumbar spinal stenosis. J Neurosurg Spine 2013; 18(4): 347355, https://doi.org/10.3171/2012.11.spine12402.

9. Kiapour A., Anderson D.G., Spenciner D.B., Ferrara L., Goel V.K. Kinematic effects of a pedicle-lengthening osteotomy for the treatment of lumbar spinal stenosis.
J Neurosurg Spine 2012; 17(4): 314-320, https://doi. org/10.3171/2012.6.spine11518.

10. Maisel W.H. Medical device regulation: an introduction for the practicing physician. Ann Intern Med 2004; 140(4): 296, https://doi.org/10.7326/0003-4819-140-4-200402170-00012.

11. Goel V.K., Ferrara L. Basic Science Symposium III: Animal Models for Orthopaedic Implant Evaluation. SAS $J$ 2008; 2(4): 195-200, https://doi.org/10.1016/s19359810(08)70039-2.

12. Demir T., Özkaya M. Mechanical testing standards of orthopedic implants. In: Korkusuz F. (editors). Musculoskeletal research and basic science. Springer; 2016; p. 61-91, https:// doi.org/10.1007/978-3-319-20777-3_5.

13. Graham J., Estes B.T. What standards can (and can't) tell us about a spinal device. SAS J 2009; 3(4): 178-183, https://doi.org/10.1016/j.esas.2009.11.001.

14. ASTM F1717-15. Standard Test Methods for Spinal Implant Constructs in a Vertebrectomy Model. ASTM International, West Conshohocken, PA; 2015, https://doi. org/10.1520/f1717-15.

15. ISO 12189:2008. Implants for surgery - Mechanical testing of implantable spinal devices - Fatigue test method for spinal implant assemblies using an anterior support. URL: https://www.iso.org/standard/39288.html.

16. La Barbera L., Galbusera F., Villa T., Costa F., Wilke H.-J. ASTM F1717 standard for the preclinical evaluation of posterior spinal fixators: can we improve it? Proceedings of the Institution of Mechanical Engineers, Part H: Journal of Engineering in Medicine 2014; 228(10): 1014-1026, https://doi. org/10.1177/0954411914554244.

17. La Barbera L., Villa T. ISO 12189 standard for the preclinical evaluation of posterior spinal stabilization devices - I: assembly procedure and validation. Proceedings of the Institution of Mechanical Engineers, Part $\mathrm{H}$ : Journal of Engineering in Medicine 2016; 230(2): 122-133, https://doi. org/10.1177/0954411915621587.

18. Wilke H., Neef P., Caimi M., Hoogland T., Claes L.E. New in vivo measurements of pressures in the intervertebral disc in daily life. Spine 1999; 24(8): 755-762, https://doi. org/10.1097/00007632-199904150-00005.

19. Gonzalez-Blohm S.A., Doulgeris J.J., Lee W.E., Shea T.M., Aghayev K., Vrionis F.D. The current testing protocols for biomechanical evaluation of lumbar spinal implants in laboratory setting: a review of the literature. Biomed Res Int 2015; 2015: 1-15, https://doi. org/10.1155/2015/506181.

20. Panjabi M.M., Brand R.A., White A.A. Threedimensional flexibility and stiffness properties of the human thoracic spine. J Biomech 1976; 9(4): 185-192, https://doi. org/10.1016/0021-9290(76)90003-8.

21. Panjabi M.M., Kato Y., Hoffman H., Cholewicki J., Krag M. A study of stiffness protocol as exemplified by testing of a burst fracture model in sagittal plane. Spine 2000; 25(21): 2748-2754, https://doi.org/10.1097/00007632-200011010-00006.

22. Panjabi M.M. Hybrid multidirectional test method to evaluate spinal adjacent-level effects. Clin Biomech 2007; 22(3): 257-265, https://doi.org/10.1016/j.clinbiomech.2006.08.006.

23. Goel V.K., Grauer J.N., Patel T.Ch., Biyani A., Sairyo K., Vishnubhotla S., Matyas A., Cowgill I., Shaw M., Long R., Dick D., Panjabi M.M., Serhan H. Effects of Charité artificial disc on the implanted and adjacent spinal segments mechanics using a hybrid testing protocol. Spine 2005; 30(24): 27552764, https://doi.org/10.1097/01.brs.0000195897.17277.67. 


\section{ADVANCED RESEARCHES}

24. Goel V.K., Winterbottom J.M. Experimental investigation of three-dimensional spine kinetics. Spine 1991; 16(8): 1000-1001, https://doi.org/10.1097/00007632199108000-00029.

25. Dreischarf M., Shirazi-Adl A., Arjmand N., Rohlmann A., Schmidt $H$. Estimation of loads on human lumbar spine: a review of in vivo and computational model studies. J Biomech 2016; 49(6): 833-45, https://doi.org/10.1016/j. jbiomech.2015.12.038.

26. Fagan M.J., Julian S., Mohsen A.M. Finite element analysis in spine research. Proceedings of the Institution of Mechanical Engineers, Part $H$ : Journal of Engineering in Medicine 2002; 216(5): 281-298, https://doi. org/10.1243/09544110260216568.

27. Jones A.C., Wilcox R.K. Finite element analysis of the spine: towards a framework of verification, validation and sensitivity analysis. Med Eng Phys 2008; 30(10): 1287-1304, https://doi.org/10.1016/j.medengphy.2008.09.006.

28. Dol' E.S., Ivanov D.V. Modelirovanie poyasnichnogo otdela pozvonochnika metodom konechnykh elementov. $V \mathrm{kn}$.: Prakticheskaya biomekhanika [Modeling of the lumbar spine by the finite element method. In: Practical biomechanics]. Saratov; 2015; p. 11-15.

29. Grauer J.N., Biyani A., Faizan A., Kiapour A., Sairyo K., Ivanov A., Ebraheim N.A., Patel T.Ch., Goel V.K. Biomechanics of two-level Charité artificial disc placement in comparison to fusion plus single-level disc placement combination. Spine J 2006; 6(6): 659-666, https://doi.org/10.1016/j. spinee.2006.03.011.

30. Ivanov A., Kiapour A., Ebraheim N., Goel V.K. Simulation of the transverse fractures of the sacrum using a finite element model of lumbar spine-pelvis segment. ASME 2008 Summer Bioengineering Conference, Parts $A$ and $B$ 2008, https://doi.org/10.1115/sbc2008-193290.

31. Parapalli B. The effects on motion and intra discal pressure after adding a dynamic stabilization device to an injured spine: a finite element based study. In: SAS8, Global Symposium on Motion Preservation Technology. Miami Beach, Florida; 2008; p. 394.

32. Kiapour A., Goel V.K. Biomechanics of a novel lumbar total motion segment preservation system: a computational and in vitro study. Orthoworld 2009; fall 2009: 86-90. 